\title{
The erratic dynamical life of black hole seeds in high-redshift galaxies
}

\author{
Hugo Pfister, ${ }^{1 \star}$ Marta Volonteri, ${ }^{1}$ Yohan Dubois, ${ }^{1}$ Massimo Dotti ${ }^{2,3}$ and Monica Colpi ${ }^{2,3}$ \\ ${ }^{1}$ Sorbonne Universités, UPMC Université Paris 06 et CNRS, UMR7095, Institut d'Astrophysique de Paris, \\ 98bis boulevard Arago, F-75014, Paris, France \\ ${ }^{2}$ Dipartimento di Fisica G. Occhialini, Università degli Studi di Milano-Bicocca, Piazza della Scienza 3, I-20126 Milano, Italy \\ ${ }^{3}$ INFN, Sezione Milano-Bicocca, Piazza della Scienza 3, I-20126 Milano, Italy
}

Accepted XXX. Received YYY; in original form ZZZ

\begin{abstract}
The dynamics of black hole seeds in high redshift galaxies is key to understand their ability to grow via accretion and to pair in close binaries during galactic mergers. To properly follow the dynamics of black holes we develop a physically motivated model to capture unresolved dynamical friction from stars, dark matter and gas. We first validate the model and then we use it to investigate the dynamics of seed black holes born at $z \sim 9$ in dwarf proto-galaxies. We perform a suite of zoom cosmological simulations with spatial resolution as high as $10 \mathrm{pc}$ and with a stellar and dark matter mass resolution of $2 \times 10^{3} \mathrm{M}_{\odot}$ and $2 \times 10^{5} \mathrm{M}_{\odot}$ respectively. We first explore the dynamics of a seed black hole in the galaxy where it is born and show that it is highly erratic if the seed mass is less than $10^{5} \mathrm{M}_{\odot}$. The dynamics is dominated by the stellar component, whose distribution is irregular and patchy, thus inducing stochasticity in the orbits: the black hole may be anywhere in the proto-galaxy. When this dwarf merges into a larger galaxy, it is paramount to simulate the process with very high spatial and mass resolution in order to correctly account for the stripping of the stellar envelope of the satellite black hole. The outcome of the encounter could be either a tight binary or, at least temporary, a wandering black hole, leading to multiple black holes in a galaxy, each inherited from a different merger.
\end{abstract}

Key words: galaxies: kinematics and dynamics - galaxies: evolution

\section{INTRODUCTION}

The high redshift Universe is the birthplace of the seeds of the supermassive black holes (BHs) observed in today's galaxy center (Kormendy \& Ho 2013a). A variety of different physical mechanisms for seed formation have been proposed (Woods et al. 2018, and references therein), but observational constraints are hampered, since the seeds are predicted to have relatively low masses $\left(10^{2}-10^{5} \mathrm{M}_{\odot}\right)$ and form at high redshift $(z>6)$, making their electromagnetic emission faint (Reines \& Comastri 2016).

The seeds build up their mass via accretion of gas and stars, or via mergers with other BHs (e.g. Volonteri et al. 2003). When BHs merge, they emit gravitational waves, and detection of such waves provides a complementary way of probing BH seeds (Sesana et al. 2007a; Barausse 2012; Ricarte \& Natarajan 2018; Hartwig et al. 2018; Dayal et al. 2018). For BHs with masses in the range $10^{4}-10^{7} \mathrm{M}_{\odot}$ the gravitational waves have frequency around $\mathrm{mHz}$, and they

^ E-mail: pfister@iap.fr are therefore primary targets for LISA, which can detect BHs with such masses out to $z>20$ (Amaro-Seoane et al. 2017).

However, before coalescing by emission of gravitational waves, which can merge BHs of $10^{4}-10^{7} \mathrm{M}_{\odot}$ in less than a Hubble time once their separation is $\sim 10^{-4}-10^{-2} \mathrm{pc}$, BHs have a long journey (Begelman et al. 1980). They are initially separated by tens of $\mathrm{kpc}$ and sit in the center of separate galaxies, which eventually merge. Then, the long process of dynamical friction (Chandrasekhar 1943) begins, driving $\mathrm{BHs}$ toward the center of the galaxy remnant, until they form a binary when their separation is pc-scale (e.g. Mayer et al. 2007; Pfister et al. 2017, and references therein). Once the binary has formed, scattering with stars (e.g. Quinlan 1996; Sesana et al. 2007b; Khan et al. 2012; Vasiliev et al. 2015), interactions with massive or circumbinary discs (e.g. Dotti et al. 2007; Haiman et al. 2009; Goicovic et al. 2016) or even three-body scattering with another incoming BH (see Bonetti et al. 2018, and references therein) are invoked to bridge the final gap to where emission of gravitational waves becomes efficient. 
Cosmological simulations are excellent tools to study the properties of $\mathrm{BH}$ evolution over cosmic time, since they can track the joint evolution of $\mathrm{BHs}$ and of the galaxies they are embedded in (Tremmel et al. 2018a). Large-volume simulations provide good statistics, having a large number of galaxies and BHs in their boxes, but lack of mass and spatial resolution means that not even the formation of $\mathrm{BH}$ binaries can be resolved. Zoom simulations can have much higher resolution, but they allow for the study of a limited number of galaxies and BHs. In this paper, we present a model to better track the dynamics of $\mathrm{BHs}$, validate it and show its limitations. We then use our model in highresolution zoomed cosmological simulations to study the yet unexplored dynamics of BHs of mass $10^{4} \mathrm{M}_{\odot}-10^{5} \mathrm{M}_{\odot}$, in a cosmological context, primary targets for the LISA observatory (Amaro-Seoane et al. 2017) .

\section{DYNAMICAL FRICTION IN NUMERICAL SIMULATIONS}

Due to their high mass, BHs attract surrounding material, gas, stars and dark matter, which create an overdensity lagging their passage. This overdensity drags and decelerates the moving BH: this phenomenon is referred to as dynamical friction (Chandrasekhar 1943; Chapon et al. 2013). To resolve the resulting force in a numerical simulation, Pfister et al. (2017) have shown that the spatial resolution, or the softening, should be smaller than the influence radius

$$
r_{\text {inf }}=\frac{\mathrm{G} M_{\bullet}}{\sigma^{2}}=1 \mathrm{pc}\left(\frac{M_{\bullet}}{10^{7} \mathrm{M}_{\odot}}\right)\left(\frac{\sigma}{200 \mathrm{~km} \mathrm{~s}^{-1}}\right)^{-2},
$$

where $M_{\bullet}$ is the mass of the $\mathrm{BH}$ and $\sigma$ is the velocity dispersion of material (gas, stars or dark matter) around the BH. This is because the typical size of the drag, partly causing dynamical friction, has a typical size of the same order as $r_{\text {inf }}$ (Colpi et al. 1999). In cosmological simulations, the typical resolution is $\sim 100 \mathrm{pc}-1 \mathrm{kpc}$, much larger than the pc-scale needed to resolve $r_{\text {inf }}$ for a $10^{7} \mathrm{M}_{\odot} \mathrm{BH}$ in a Milky-Way like galaxy. Therefore, we must remove by hand the momentum that a $\mathrm{BH}$ would lose through dynamical friction if we were able to resolve the phenomenon. In this section we first describe how we implement unresolved dynamical friction in the adaptive mesh refinement code Ramses (Teyssier 2002) for collisionless particles (stars and dark matter); the code already includes a correction for dynamical friction from gas (Dubois et al. 2012).

We follow an approach similar to Tremmel et al. (2015), although we include not only the contribution to dynamical friction from slow moving particles but also from fast moving particles, which can play an important role when the density profile becomes shallow (Antonini \& Merritt 2012; Dosopoulou \& Antonini 2017).

We measure all the quantities needed to estimate dynamical friction in a sphere $\mathcal{S}$ centered on the $\mathrm{BH}$ with a radius $4 \Delta x$, where $\Delta x$ is corresponds to the minimum grid size. We chose $\mathcal{S}$ to be consistent with the already existing implementation for gas accretion, feedback and dynamical friction (Dubois et al. 2012).

We report here Eq. (30) from Chandrasekhar (1943). This gives an analytical estimate of the amount of momentum that must be removed to BHs due to dynamical friction:

$$
\begin{aligned}
\vec{a}_{\mathrm{DF}} & =-4 \pi \mathrm{G}^{2} M \bullet \frac{\vec{v}_{\bullet}}{v_{\bullet}^{3}}\left(\ln \Lambda \int_{0}^{v_{\bullet}} 4 \pi v^{2} f(v) d v+\ldots\right. \\
& \left.\ldots \int_{v_{\bullet}}^{\infty} 4 \pi v^{2} f(v)\left[\ln \left(\frac{v+v_{\bullet}}{v-v_{\bullet}}\right)-2 \frac{v_{\bullet}}{v}\right] d v\right),
\end{aligned}
$$

where we denote as $M_{\bullet}$ the mass of the $\mathrm{BH}$, as $\vec{v}_{\bullet}$ (with magnitude $v_{\bullet}$ ) the relative velocity of the $\mathrm{BH}$ with respect to the velocity of the background, and $\tilde{\vec{v}}$ defined below in Eq. (4); $\ln \Lambda=\ln \left(4 \Delta x / r_{\text {def }}\right)$ is the Coulomb logarithm (this expression is justified below); and $f$ is the distribution function:

$$
4 \pi v^{2} f(v)=\frac{3}{256 \pi \Delta x^{3}} \sum_{i \in \mathcal{S}} m_{i} \delta\left(v_{i}-v\right) .
$$

Here $\vec{v}_{i}$ (with magnitude $v_{i}$ ) is the relative velocity of particle $i$ with respect to the velocity of the background, $m_{i}$ is the mass of particle $i$ and $\delta$ is the Dirac function.

The velocity of the background, $\tilde{\vec{v}}$, is simply the massweighted velocity of all particles (except the $\mathrm{BH}$ particle) enclosed in $\mathcal{S}$ :

$$
\tilde{\vec{v}}=\frac{1}{M} \sum_{i \in \mathcal{S}} \vec{v}_{i} m_{i},
$$

where $M$ is the total mass enclosed in $\mathcal{S}$. We stress here that the background velocity is computed for stars and dark matter separately, the reason is that dynamical friction assumes particles with similar masses, which is a reasonable assumptions if we consider an assembly of stars, and an assembly of dark matter particles, but not if we consider stars and dark matter particles together. Therefore we compute the contribution from dark matter, $\vec{a}_{\mathrm{DF}, \mathrm{DM}}$, and stars, $\vec{a}_{\mathrm{DF}, \star}$ separately.

We justify here the expression above for the Coulomb logarithm $\ln \Lambda=\ln \left(4 \Delta x / r_{\text {def }}\right)$. In the classical derivation of dynamical friction (Chandrasekhar 1943) the Coulomb logarithm represents the ratio between the "minimum" and "maximum" impact parameters that affect the velocity change. The minimum impact parameter represents that required to have a deflection of $90^{\circ}$, which in the Keplerian case is the deflection radius:

$$
\begin{aligned}
r_{\text {def }} & =\mathrm{G} M_{\bullet} / v_{\bullet}^{2} \\
& \simeq r_{\text {inf }}
\end{aligned}
$$

while the maximum impact parameter is the distance at which the stellar density becomes sufficiently "smaller" than around the $\mathrm{BH}$ to become insignificant in modifying its velocity. In our case, gravity is computed self-consistently by the code outside $\mathcal{S}$; therefore, the integration must be stopped at $4 \Delta x$ if we do not want to double count dynamical friction. This naturally leads to $\ln \Lambda=\ln \left(4 \Delta x / r_{\text {def }}\right)$. Furthermore, as explained in Beckmann et al. (2017), using subgrid models when resolution is sufficient to account for dynamical friction can lead to incorrect results. For this reason, when $4 \Delta x \leq r_{\text {def }}$, we set $\vec{a}_{\mathrm{DF}}$ to 0 .

\section{ADDITIONAL PHYSICS: GALAXIES AND BLACK HOLES}

Ramses follows the evolution of the gas using the secondorder MUSCL-Hancock scheme for the Euler equations. The 
approximate Harten-Lax-Van Leer Contact (Toro 1997) Riemann solver with a MinMod total variation diminishing scheme to reconstruct the interpolated variables from their cell-centered values is used to compute the unsplit Godunov fluxes at cell interfaces. An equation of state of perfect gas composed of monoatomic particles with adiabatic index $\gamma=5 / 3$ is assumed to close the full set of fluid equations. Collisionless particles (dark matter, stellar and $\mathrm{BH}$ particles) are evolved using a particle-mesh solver with a cloud-in-cell interpolation. The size of the cloud-in-cell interpolation is that of the local cell for BHs and stars, however, dark matter particles can only project their mass on the grid down to a minimum cell size of $\Delta x_{\mathrm{DM}}>\Delta x$ (as these particles are usually larger in mass than stars or gas, we smooth their mass distribution to reduce their contribution to shot noise). When cloud-in-cell interpolation is used, therefore, even if the mass of the dark matter particle is larger than the $\mathrm{BH}$ mass, since the dark matter distribution is smoothed, scattering off dark matter particles becomes unimportant.

Gas is allowed to cool by hydrogen and helium with a contribution from metals using cooling curves from Sutherland \& Dopita (1993) for temperatures above $10^{4} \mathrm{~K}$. For gas below $10^{4} \mathrm{~K}$ and down to our minimum temperature of $10 \mathrm{~K}$, we use the fitting functions of Rosen \& Bregman (1995).

Star formation is stochastically sampled from a random Poisson distribution (Rasera \& Teyssier 2006): at each timestep $\Delta t$, in each cell of size $\Delta x$ containing a gas mass $M_{\text {gas }}$, the mass of newly formed stars, $M_{\star \text {,new }}$, follows a Schmidt law:

$$
M_{\star, \text { new }}=\epsilon \frac{M_{\mathrm{gas}}}{t_{\mathrm{ff}}} \Delta t,
$$

where $t_{\mathrm{ff}}=\sqrt{3 \pi / 32 \mathrm{G} \rho_{\text {gas }}}$ is the free-fall time, $\rho_{\text {gas }}=M_{\text {gas }} / \Delta x^{3}$ is the gas density in the cell, and $\epsilon$ depends on the local turbulence of the gas, as detailed in Trebitsch et al. (2018).

For the feedback of supernovae, we use the mechanical feedback described in Kimm \& Cen (2014), in which star particles older than $5 \mathrm{Myr}$ release $\eta_{\mathrm{SN}} \times 10^{50} \mathrm{erg} / \mathrm{M}_{\odot}$, where $\eta_{\mathrm{SN}}=0.2$. The amount of energy and momentum deposited depends on local properties of the gas (density and metallicity) so that it captures either the Sedov or the supernovaeow-plough expansion phase of the explosion.

We use the model of BHs described in Dubois et al. (2012), where accretion is computed using the Bondi-HoyleLittleton formalism capped at the Eddington luminosity. AGN feedback consists of a dual-mode approach, where thermal energy, corresponding to $15 \%$ of the bolometric luminosity (with radiative efficiency of $\epsilon_{\mathrm{r}}=0.1$ ), is injected at high accretion rates (luminosity above 0.01 the Eddington luminosity); otherwise feedback is modeled with a bipolar jet with a velocity of $10^{4} \mathrm{~km} \mathrm{~s}^{-1}$ and an efficiency of $100 \%$. We slightly modify the implementation of $\mathrm{BH}$ dynamics: in the original Ramses version, the mass of the $\mathrm{BH}$ is deposited onto the so-called "cloud" particles, which uniformly pave a sphere of $4 \Delta x$ radius on a grid of $\Delta x / 2$ inter-cloud distance. This has the effect of smoothing the density, and therefore, when two BHs pass close by, their potential is shallower than it should be, and this delays the formation of the binary. We simply deposit all the mass of BHs onto their central cloud particle and then perform the cloud-in-cell to obtain more accurate dynamics, while using the rest of cloud particles to compute the Bondi-Hoyle-Littleton accretion rate onto the $\mathrm{BH}$.

We include the dynamical friction implementation as described in Section 2 when necessary, and dynamical friction from gas was already included (Dubois et al. 2014a) using Eq. (12) from Ostriker (1999). Two BHs are allowed to merge when they are separated by less than $4 \Delta x$ and the kinetic energy of the binary is lower than the gravitational energy.

Due to our inability to resolve the cold and dense regions of the interstellar medium, gas dynamical friction and gas accretion, which depend linearly on the gas density, $\rho_{\text {gas }}$ can be underestimated in simulations. To correct for this lack of resolution, Booth \& Schaye (2009); Dubois et al. (2014a) compute the expressions obtained analytically (Chandrasekhar gas dynamical friction and Bondi accretion) and boost them by:

$$
\text { boost }=\left(\frac{\rho_{\mathrm{gas}}}{\rho_{\mathrm{th}}}\right)^{\xi},
$$

where $\rho_{\text {th }}$ is a free parameter, similar to that used for star formation, which is linked to the Jeans length and depends on resolution. This parameter is typically calibrated via the phase diagram of gas, and it is $\sim 1 \mathrm{amu} \mathrm{cm}^{-3}$ for $\Delta x=100 \mathrm{pc}$ and $\sim 50 \mathrm{amu} \mathrm{cm}^{-3}$ for $\Delta x=10 \mathrm{pc}$. $\xi$ can differ for accretion and dynamical friction, for the rest of the paper, we will use $\xi=\alpha$ when we relate to boosting accretion, and $\xi=\beta$ when we refer to gas dynamical friction. Booth \& Schaye (2009) performed a parameter study and found that $\alpha=2$ is the optimal value to recover the $\mathrm{BH}$ mass - galaxy mass relation (Kormendy \& Ho 2013b). Although the expression of these boosts is not physically motivated, they have been proven to give excellent match with observations, as shown by large cosmological simulations (Dubois et al. 2014b). For this work we will either use $\xi=2$ either $\xi=0$ (no boost), and in Section 4.2 we also vary $\rho_{\text {th }}$ within an order of magnitude of the typical value expected for the chosen resolution. We briefly explore the effects of the boost in $\S 4.2$.

\section{VALIDATION OF THE DYNAMICAL FRICTION IMPLEMENTATION}

\subsection{Isolated dark matter halo}

In order to compare the dynamical friction timescale with analytical estimates (Lacey \& Cole 1993; Colpi et al. 1999; Taffoni et al. 2003), we test our implementation following the dynamics of a BH moving in a dark matter halo.

The dark matter halo, initialized with DICE (Perret 2016), follows a Navarro, Frank and White (NFW, Navarro et al. 1997) profile with a total virial mass $M_{\mathrm{vir}}=2 \times 10^{11} \mathrm{M}_{\odot}$, a concentration parameter of 4 and a virial radius $R_{\mathrm{vir}}=$ $45 \mathrm{kpc}$, typical of redshift 3 . We set the total spin parameter to 0.04 consistent with the average spin parameter of cosmological dark matter halos (Bullock et al. 2001), which only mildly evolves between $z=3$ and today (Muñoz-Cuartas et al. 2011; Ahn et al. 2014). The BH mass is set to $10^{8} \mathrm{M}_{\odot}$, it is initially $5 \mathrm{kpc}$ away from the center with a tangential velocity of $57 \mathrm{~km} \mathrm{~s}^{-1}$, corresponding to to $50 \%$ of the circular velocity. In the simulation, the influence radius varies between 10 and 100 pc: it is at best resolved by 2 cell elements, therefore dynamics is generally not treated properly 
and dynamical friction must be added ad hoc with our subgrid model when necessary.

As done in Tremmel et al. (2015), we can estimate the goodness of the method by comparing it to the analytical estimate of the "sinking time", $\tau_{\mathrm{DF}}$, defined as the time it will take for a satellite to sink to a target, using Eq. (12) from Taffoni et al. (2003):

$$
\begin{aligned}
\tau_{\mathrm{DF}}= & 0.6 \frac{r_{c}^{2} v_{c}}{\mathrm{G} M_{s}} \log ^{-1}\left(1+\frac{M_{\mathrm{vir}}}{M_{s}}\right)\left(\frac{J}{J_{c}}\right)^{\alpha} \\
\simeq & 1.4 \mathrm{Gyr}\left(\frac{r_{c}}{100 \mathrm{pc}}\right)^{2}\left(\frac{v_{c}}{10 \mathrm{~km} \mathrm{~s}^{-1}}\right)\left(\frac{10^{4} \mathrm{M}_{\odot}}{M_{s}}\right) \\
& \log ^{-1}\left(1+\frac{M_{\mathrm{vir}}}{M_{s}}\right)\left(\frac{J}{J_{c}}\right)^{\alpha},
\end{aligned}
$$

where $M_{\mathrm{vir}}$ is the virial mass of the target, $v_{c}$ is the circular velocity at the virial radius, $\mathrm{G}$ the gravitational constant, $r_{c}$ is the radius at which a test particle moving in the potential of the target has the same energy as the satellite, $M_{S}$ is the mass of the satellite, $J$ is the specific angular momentum of the satellite in the frame of the target, $J_{c}$ is the specific angular momentum of circular orbit at $r_{c}$ and $\alpha$ depends on $M_{S}, M_{\mathrm{vir}}, R_{\mathrm{vir}}$ and $r_{c}$ and is given by Eq. (15) from Taffoni et al. (2003). In this case, the target is the halo and the satellite is the BH. Using this approach, we find that the $\mathrm{BH}$ should sink in the potential well of the halo in $600 \mathrm{Myr}$.

We perform two simulations which only differ by the presence (PD), or not (NoDrag), of dynamical friction onto the BH using our subgrid model. In both cases, the size of the box is $100 \mathrm{kpc}$, slightly larger than $2 R_{\mathrm{vir}}$ and we allow refinement from levels 7 to 11 , leading to a maximum physical resolution of $\Delta x=\Delta x_{\mathrm{DM}}=50 \mathrm{pc}$, similar to what simulations reach in cosmological zooms (Dubois et al. 2014a). The refinement is done using a quasi-Lagrangian criterion: a cell is refined if its mass exceeds $8 \times m_{\mathrm{DM}}$, where $m_{\mathrm{DM}}$ is the mass of dark matter particles, and we refine at maximum level up to $4 \Delta x$ around the BH. We set the mass of dark matter particles to $10^{5} \mathrm{M}_{\odot}$, in good agreement with the value suggested by Power et al. $(2003): m_{\mathrm{DM}}=M_{\text {vir }}\left(R_{\text {vir }} / \Delta x\right)^{-2} \sim 2 \times 10^{5} \mathrm{M}_{\odot}$.

We show in Fig. 1, for both simulations, the distance between the $\mathrm{BH}$ and the center of the halo; we also include for comparison the analytical estimate given by Eq. (10). The result is quite clear and in agreement with Tremmel et al. (2015): adding unresolved dynamical friction contributes to recover sinking times estimated analytically. In the following section, we set in a more realistic problem where a $\mathrm{BH}$ sinks in a galaxy including not only dark matter but also gas, stars and many associated processes (cooling, star formation, supernovae feedback).

\subsection{Isolated galaxy}

We run a suite of simulations (see Table 1) of a BH sinking in the potential well of an idealized isolated galaxy. Our suite contains low-resolution $\left(\Delta x=\Delta x_{\mathrm{DM}}=50 \mathrm{pc}\right)$ simulations, similar to what high-resolution zoomed cosmological simulations can reach today. Thus it is a good test to see how our implementation will act in this context. Contrary to the dark matter halo case, we do not have analytical estimates to provide a benchmark. To overcome this issue, we run a high resolution test $\left(\Delta x=\Delta x_{\mathrm{DM}}=1 \mathrm{pc}\right)$ to perform the comparison. The setup is chosen such that, with

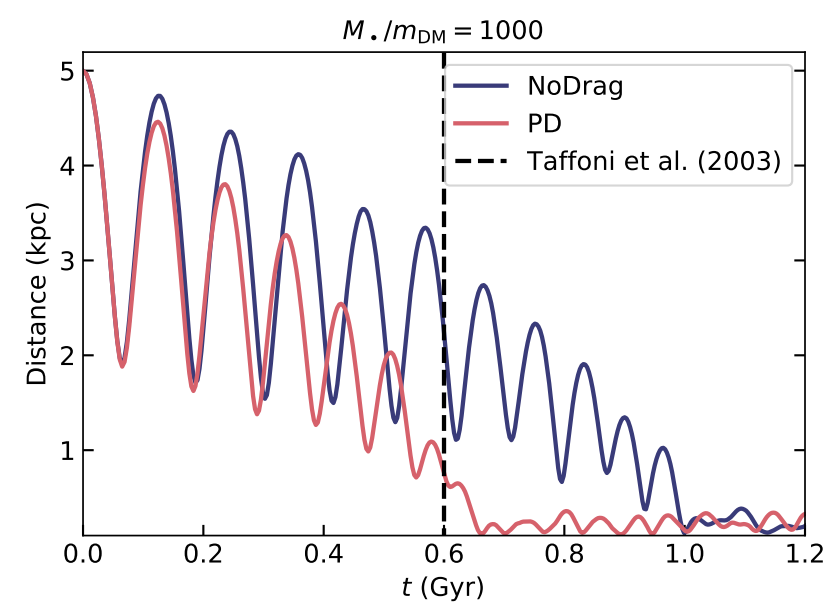

Figure 1. Distance of the BH to the center of the halo as a function of time. The vertical dashed line is the analytical estimate of the total sinking time from Taffoni et al. (2003). With our prescription, the sinking time is much shorter than without and in very good agreement with the analytical estimate. The resolution in these simulations is $50 \mathrm{pc}$ and the $\mathrm{BH}$ to dark matter mass particle ratio set equal to 1000 . See section 4.1 for details.

\begin{tabular}{lcccc}
\hline Name & Dynamical friction & $\begin{array}{c}\rho_{\mathrm{th}}^{[1]} \\
\mathrm{amu} \mathrm{cc}\end{array}$ & $\beta^{[2]}$ & $\begin{array}{c}\Delta x^{[3]} \\
\mathrm{pc}\end{array}$ \\
\hline \hline NoDrag & none & $\mathrm{X}$ & $\mathrm{X}$ & 50 \\
GnB_nPD & gas (no boost) & $\mathrm{X}$ & 0 & 50 \\
GB0.1_nPD & gas (boost) & 0.1 & 2 & 50 \\
GB1_nPD & gas (boost) & 1 & 2 & 50 \\
GB15_nPD & gas (boost) & 15 & 2 & 50 \\
GnB_PD & gas (no boost)+stars+dark matter & $\mathrm{X}$ & 0 & 50 \\
HR & none & $\mathrm{X}$ & $\mathrm{X}$ & 0.76 \\
\hline
\end{tabular}

Table 1. Different simulations performed for the isolated galaxy test, with their name and the use, or not, of our new model. [1-2] Typical density and exponent that we used to boost gas friction, following eq. (8). [3] Resolution of the simulation.

$50 \mathrm{pc}$ resolution, during the sinking, the deflection radius, as defined in Eq. (5), is not always resolved (see Fig. 2). In this case, dynamics is not properly treated and dynamical friction must be added ad hoc with our subgrid model. Conversely, with $1 \mathrm{pc}$ resolution the deflection radius is always resolved during the sinking and dynamical friction is well captured by the gravity solver of Ramses, thus providing the correct dynamics.

We initialize with DICE an ideal galaxy at redshift 3 with a total virial mass of $2 \times 10^{11} \mathrm{M}_{\odot}$ and a spin parameter of 0.04 . The galaxy is composed of four components.

- A dark matter halo with a mass of $1.95 \times 10^{11} \mathrm{M}_{\odot}$, slightly lighter than in $\S 4$. I It has a virial radius of $45 \mathrm{kpc}$ and the density follows a NFW profile with a concentration parameter of 4 .

- A gas disk with a total mass of $2.4 \times 10^{9} \mathrm{M}_{\odot}$. The density follows an exponential disk + sech- $z$ profile with a scale radius of $1.28 \mathrm{kpc}$ and an aspect ratio of $1: 10$. We impose 
an initial constant absolute metallicity and temperature of $10^{-3}$ and $10^{5} \mathrm{~K}$, respectively.

- A stellar disk with a total mass of $1.6 \times 10^{9} \mathrm{M}_{\odot}$. The density follows an exponential disk + sech- $z$ profile with a scale radius of $1.28 \mathrm{kpc}$ and an aspect ratio of $1: 10$. We impose an initial constant absolute metallicity of $10^{-3}$. Additionally, to avoid unphysical initial starbursts regularly found in ideal simulations (Capelo et al. 2015), we give an age distribution to stellar particles to mimic a $5 \mathrm{M}_{\odot} \mathrm{yr}^{-1}$ star formation rate.

- A stellar bulge with a total mass of $8 \times 10^{8} \mathrm{M}_{\odot}$. The density follows a Hernquist profile (Hernquist 1990) with a scale radius of $0.128 \mathrm{kpc}$. We impose a constant absolute metallicity of $2 \times 10^{-4}$ ( 5 times smaller than in the disk to mimic the older age of stars in the bulge). Similarly, we give an age to stellar particles to mimic a $0.5 \mathrm{M}_{\odot} \mathrm{yr}^{-1}$ star formation rate.

In the low-resolution simulations (50 pc), the mass of dark matter particles is set to $10^{6} \mathrm{M}_{\odot}$ and that of star particles to $2 \times 10^{4} \mathrm{M}_{\odot}$. In the high resolution simulations (1 pc) the mass of dark matter particles is set to $5 \times 10^{4} \mathrm{M}_{\odot}$ and that of star particles to $2 \times 10^{3} \mathrm{M}_{\odot}$. In both cases the size of the box is $100 \mathrm{kpc}$ and we allow for refinement from levels 7 to 11 in the low resolution simulations and from 7 to 17 in the high resolution one, refining the mesh when $M_{\mathrm{DM}}^{\text {cell }}+10 M_{b}^{\text {cell }} \geq 8 m_{\mathrm{DM}}$, where $M_{\mathrm{DM}}^{\text {cell }}$ and $M_{b}^{\text {cell }}$ are, respectively, the mass of dark matter and baryons in the cell. Maximum refinement is enforced within $4 \Delta x$ around the BH.

After initializing this galaxy, we switch on cooling, star formation, supernovae feedback (see $\S 3$ ) and let the galaxy relax for 100 Myr. At that point, a $\mathrm{BH}$ with mass $10^{7} \mathrm{M}_{\odot}$ is placed in the $z=0$ plane, $1 \mathrm{kpc}$ from the center and with a tangential velocity of $21 \mathrm{~km} / \mathrm{s}$, corresponding to $30 \%$ of the circular velocity. Accretion and feedback from the BH are not included in order to keep the BH mass constant and isolate the effects of dynamical friction. We include dynamical friction with different implementations: from collisionless particles and gas without boost (i.e. no free parameters), or only from gas, with or without a boost factor. The simulation properties and set-up are summarized in Table 1.

We show in Fig. 3, for all our simulations, the distance between the $\mathrm{BH}$ and the center of the galaxy as a function of time. We first stress the difference between low resolution simulations with gravity only, i.e. without including the dynamical friction model (NoDrag, blue line), and the simulations at high resolution where the deflection radius is resolved (HR, black line). In agreement with the results of Pfister et al. (2017), resolving at least the deflection radius is mandatory to properly capture the dynamics of the $\mathrm{BH}$ in the dynamical friction phase.

We now compare simulations where we vary $\rho_{\text {th }}$ (GB0.1_nPD, GB1_nPD, GB15_nPD) but we do not include dynamical friction from stars and dark matter. As expected, the lower $\rho_{\text {th }}$, the larger the boost, the faster the BH sinks. The choice of $\rho_{\text {th }}$ must be performed accurately: if $\rho_{\text {th }}$ is too low, BHs can get caught in a passing clump and either follow the clump outside the galaxy center, or remain artificially in a dense environment where accretion is triggered, resulting in an overestimate of the mass of BHs. If $\rho_{\text {th }}$ is too high, instead, the correction to dynamical friction is insufficient and the orbital decay is delayed. In this particular case, $\rho_{\text {th }}$ between 0.1 and $1 \mathrm{amu} \mathrm{cm}^{-3}$ is the best value to recover the

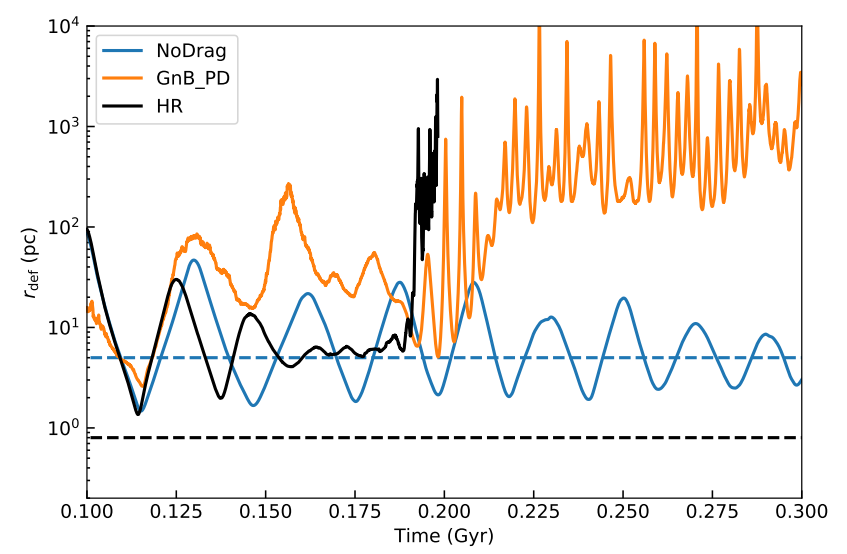

Figure 2. Deflection radius (solid line) and resolution of the different simulations (dashed lines) of idealized isolated galaxies. In the low-resolution case the deflection radius is not always resolved, leading to incorrect dynamics of the $\mathrm{BH}$ and the need to add unresolved dynamical friction. In the high resolution run the deflection radius is always resolved and dynamical friction is selfconsistently captured by the gravity solver. All quantities shown as a function of time.

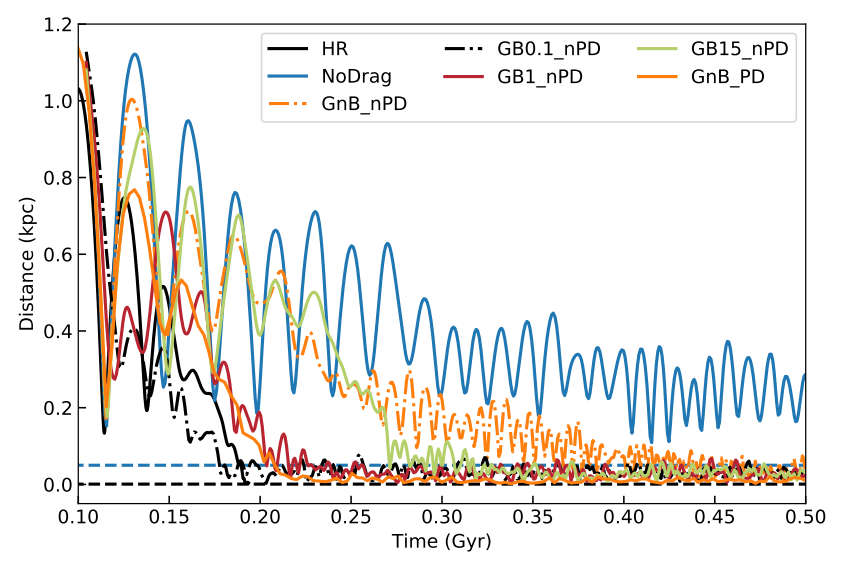

Figure 3. Distance of the $\mathrm{BH}$ to the center of the galaxy as a function of time and resolutions of the different simulations (dashed lines). The good behavior of our model is confirmed by the agreement between the solid orange curve (low resolution, use of our model) and the solid black one (high resolution). See section 4.2 for details.

high resolution results, but the exact value may depend on additional factors such as the gas fraction ( $50 \%$ in our case).

We finish with the simulation where the influence radius is not always resolved, but in which we include sub-grid dynamical friction from stars, dark matter and gas (without any boost) following our implementation (GnB_PD, orange line). This implementation does not contain any free parameters and avoids the arbitrary choice of $\rho_{\text {th }}$. This simulation is in excellent agreement with the high resolution simulation (HR, thin black line), confirming the good behavior of our model in a realistic, although idealized, galaxy. 


\begin{tabular}{lccc}
\hline$M_{\bullet} / m_{\mathrm{DM}}$ & $\begin{array}{c}d_{0} \\
\mathrm{kpc}\end{array}$ & $\begin{array}{c}v_{0} \\
\mathrm{~km} \mathrm{~s}^{-1}\end{array}$ & $\begin{array}{c}\tau_{\mathrm{DF}} \\
\mathrm{Gyr}\end{array}$ \\
\hline \hline 10 & 2 & 8.2 & 2.38 \\
1 & 1 & 6 & 5.26 \\
\hline
\end{tabular}

Table 2. Different simulations we perform to test the limits of our model in terms of particle mass ratio. We indicate the different mass ratio between the $\mathrm{BH}$ and dark matter particles, the initial distance of the $\mathrm{BH}$ from the center of the halo, the initial velocity of the $\mathrm{BH}$ and the analytical estimate for the time the $\mathrm{BH}$ should take to reach the center of the halo from Taffoni et al. (2003). In all cases, we run a simulation with (PD) and without (NoDrag) our model.

\subsection{Limits of the model: low mass black holes}

In this section we explore the limits of our implementation when a BH has a mass so low that 2-body interactions with star and dark matter particles significantly perturb its dynamics.

We run simulations similar to those described in $\S 4.1$ but decreasing the mass of the $\mathrm{BH}$ down to the mass of dark matter particles $\left(m_{\mathrm{DM}}=10^{5} \mathrm{M}_{\odot}\right)$. To contain computational costs, we also change the orbital parameters of the BH such as the analytical estimates from Taffoni et al. (2003), $\tau_{\mathrm{DF}}$, remains a few Gyrs. We list the parameters of the simulations in Table 2.

We show in Fig. 4 the distance of the $\mathrm{BH}$ to the center of the halo as a function of time. It is clear that our model works very well when BHs have a mass larger than 10 times the mass of particles causing dynamical friction. If the mass of the $\mathrm{BH}$ is similar to that of particles causing dynamical friction, however, it is scattered through 2-body interactions and the model becomes less reliable, as also noted by Tremmel et al. (2015).

In $\S 4.2$ and $\S 5.1$, the mass of dark matter particles is larger than that of BHs. However, we use cloud-in-cell interpolation to smooth the dark matter distribution, and we ensure that the mass of star particles, which are the main source of dynamical friction, is lower than the mass of BHs.

\section{COSMOLOGICAL SIMULATIONS}

\section{$5.1 \quad$ Set-up}

We now move to the full cosmological context, endeavoring to study the dynamical behavior of seed BHs in high-redshift galaxies. We run a suite of cosmological simulations, with the code Ramses. We zoom-in on one halo using different prescriptions for the dynamics of BHs. As we are interested in understanding the evolution of $\mathrm{BHs}$ in typical galaxies, we chose a halo with a minor/major merger rate comparable to the mean evolution obtained by Fakhouri et al. (2010) in this mass range. The physics is similar to that of the simulations described in $\S 3$ but for the refinement strategy: we refine if $M_{\mathrm{DM}}^{\text {cell }}+\left(\Omega_{m} / \Omega_{b}-1\right) M_{b}^{\text {cell }} \geq 8 m_{\mathrm{DM}}$, where $M_{\mathrm{DM}}$ and $M_{b}^{\text {cell }}$ are, respectively, the mass of dark matter and baryons in the cell, and $\Omega_{m}$ and $\Omega_{b}$ are the total matter and baryon density. The minimum cell size, $\Delta x$ is kept roughly constant in proper physical size with redshift: an additional level of refinement is added every time the expansion factor, $a_{\exp }$, decreases
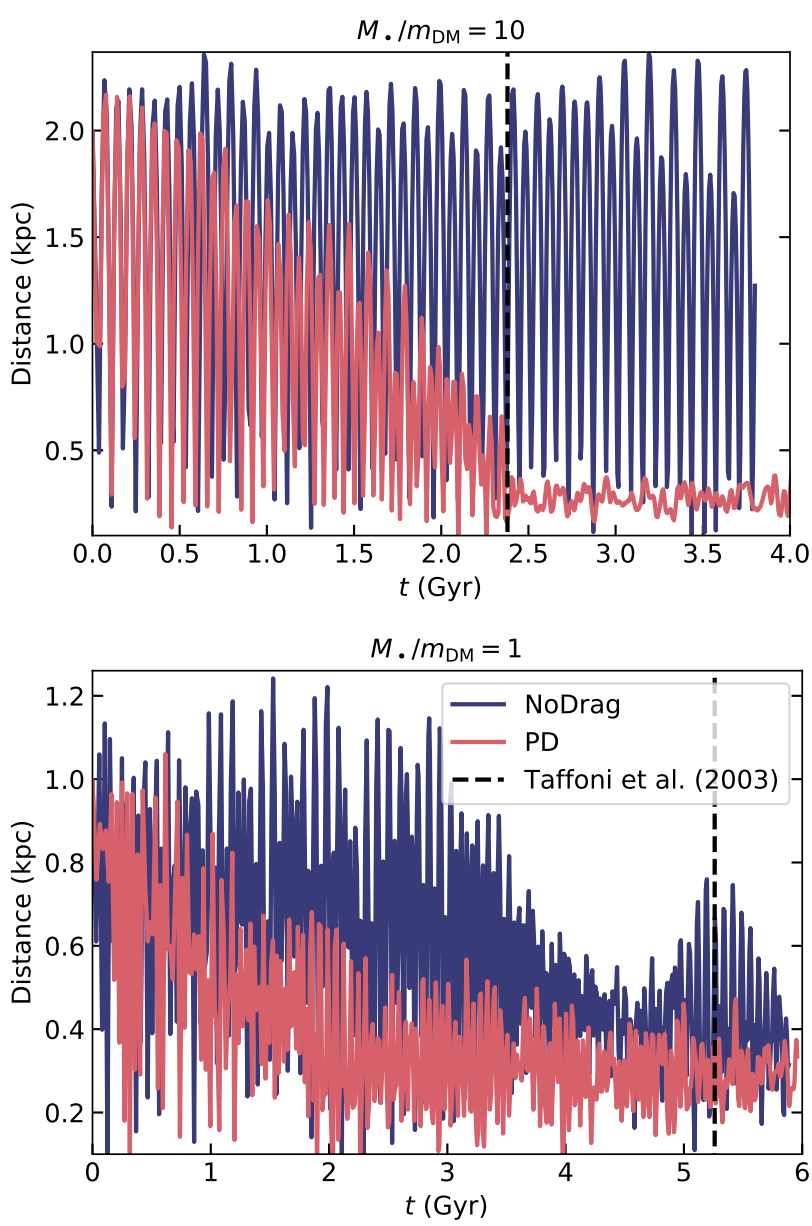

Figure 4. Different simulations performed to test the effect of reducing the mass ratio between the $\mathrm{BH}$ and dark matter particles. We indicate the use (PD) or not (NoDrag) of our prescription for dynamical friction. If the $\mathrm{BH}$ mass is similar to the dark matter particle mass, the efficacy of the model becomes limited. See section 4.3 for details.

by a factor of two, such that the maximum level, $l_{\max }$, is reached at $a_{\exp }=0.8$. For simplicity, we further assume that $\Delta x=L_{\mathrm{box}} / 2^{l_{\max }}$, where $L_{\mathrm{box}}$ is the size of the box at redshift 0 . Concerning the subgrid physics of BHs (see $\S 3$ ) we use $\beta=2$ to boost accretion, gas friction is not boosted $(\alpha=0)$ and the value of $\rho_{t h}$ depends on resolution. The specifications of each simulations are described in Table 3.

\subsubsection{Initial conditions}

We assume a $\Lambda$ CDM cosmology with total matter density $\Omega_{m}=0.3089$, baryon density $\Omega_{b}=0.0486$, dark energy density $\Omega_{\Lambda}=0.6911$, amplitude of the matter power spectrum $\sigma_{8}=0.8159, n_{s}=0.9667$ spectral index and Hubble constant $H_{0}=67.74 \mathrm{~km} \mathrm{~s}^{-1} \mathrm{Mpc}^{-1}$ consistent with the Planck data (Planck Collaboration et al. 2016). The initial conditions are produced with MUSIC (Hahn \& Abel 2013). The box size of the simulations is $L_{\mathrm{box}}=73.8 \mathrm{Mpc}$, with a coarse grid of $256^{3}$ dark matter particles corresponding to a dark matter mass resolution of $m_{\mathrm{DM}}$, coarse $=3 \times 10^{9} \mathrm{M}_{\odot}$. A high-resolution region is defined around a halo of $M_{\mathrm{vir}}=10^{12} \mathrm{M}_{\odot}$ at $z=2$ 


\begin{tabular}{lccccccc}
\hline Name & $\begin{array}{c}\text { Particle } \\
\text { dynamical friction }\end{array}$ & $\begin{array}{c}\rho_{\mathrm{th}}^{[1]} \\
\mathrm{amu} \mathrm{cc}\end{array}$ & $\begin{array}{c}\Delta x^{[2]} \\
\mathrm{pc}\end{array}$ & $\begin{array}{c}\Delta x_{\mathrm{DM}}^{[3]} \\
\mathrm{pc}\end{array}$ & $\begin{array}{c}m_{\star}^{[4]} \\
\mathrm{M}_{\odot}\end{array}$ & $\begin{array}{c}m_{\mathrm{DM}}^{[5]} \\
\mathrm{M}_{\odot}\end{array}$ & $\begin{array}{c}M_{\odot}^{[6]} \\
\mathrm{M}_{\odot}\end{array}$ \\
\hline \hline & & & & & & & \\
LR_PD_BH1e4 & $\boldsymbol{V}$ & 5 & 72 & 2300 & $2 \times 10^{4}$ & $2 \times 10^{6}$ & $10^{4}$ \\
MR_PD_BH1e4 & $\boldsymbol{V}$ & 10 & 36 & 2300 & $2 \times 10^{4}$ & $2 \times 10^{6}$ & $10^{4}$ \\
HR_PD_BH1e4 & $\boldsymbol{X}$ & 50 & 9 & 572 & $2 \times 10^{3}$ & $2 \times 10^{5}$ & $10^{4}$ \\
HR_nPD_BH1e4 & $\mathbb{N}$ & 50 & 9 & 572 & $2 \times 10^{3}$ & $2 \times 10^{5}$ & $10^{4}$ \\
HR_PD_BH1e5 & $\boldsymbol{X}$ & 50 & 9 & 572 & $2 \times 10^{3}$ & $2 \times 10^{5}$ & $10^{5}$ \\
HR_nPD_BH1e5 & 50 & 9 & 572 & $2 \times 10^{3}$ & $2 \times 10^{5}$ & $10^{5}$ \\
\hline
\end{tabular}

Table 3. Properties of the suite of cosmological simulations performed. [1] Typical density and exponent that we used to boost accretion, following Eq. (8). [2-5] Spatial/mass resolution of the simulation. [6] Seed mass of BHs.

that contains only high-resolution dark matter particles (see Table 2 for the mass of high-resolution dark matter particles in each simulation) within $2 r_{\text {vir }}\left(r_{\text {vir }}=100 \mathrm{kpc}\right)$. The halo is a progenitor of a group of galaxies whose mass is $M_{\mathrm{vir}}=7 \times 10^{12} \mathrm{M}_{\odot}$ at $z=0$.

\subsubsection{Finding halos and galaxies}

We construct catalogues of haloes and galaxies using the AdaptaHOP halo finder (Aubert et al. 2004), which uses an SPH-like kernel to compute densities at the location of each particle and partitions the ensemble of particles into subhaloes based on saddle points in the density field. Haloes contain at least 200 dark matter particles. Galaxies are identified in the same way, and contain at least 200 stellar particles. We then construct a merger tree for halos and galaxies with TreeMaker (Tweed et al. 2009).

\subsubsection{Estimate of the sinking time}

We consider once again the "sinking time", $\tau_{\mathrm{DF}}$, defined as the time it takes for a satellite to sink to a target, using Eq. 10. To compute $\tau_{\mathrm{DF}}$ for a $\mathrm{BH}$ in its own galaxy $(\$ 5.2)$, we consider that the satellite is the $\mathrm{BH}$, for which we have the dynamical properties, and we consider that the target is the galaxy, for which we compute the different properties with the halo finder.

To compute $\tau_{\mathrm{DF}}$ for a $\mathrm{BH}$ during a galaxy merger ( $(5.3)$, we have to take into account that $M_{S}$ evolves. Initially, the $\mathrm{BH}$ is surrounded by its own galaxy, which is itself surrounded by a halo, and it is the system $\mathrm{BH}+$ galaxy+halo that undergoes dynamical friction. Therefore, we must match BHs to galaxies and galaxies to halos to have the corrected satellite mass, i.e. $M_{S}$ is similar to the mass of the halo.

In a second phase, the dark matter halo and outer stellar layers of the secondary galaxy disperse into that of the primary, and the BH remains surrounded only by a fraction of the initial stellar mass, and we identify the evolving $M_{S}$ via the halo finder. Finally, the $\mathrm{BH}$ remains naked, and $M_{S}$ is the BH mass. To give an order of magnitude for this final phase, in the early universe, where galaxies have velocity dispersion as small as tens of $\mathrm{km} \mathrm{s}^{-1}$, unless the $\mathrm{BH}$ is very massive $\left(\gtrsim 10^{5} \mathrm{M}_{\odot}\right)$, or surrounded by a bound dense stellar cluster, acting as if $M_{S}$ is larger, the sinking time is longer than Gyrs if the distance to the center if larger than $\sim 100 \mathrm{pc}$, which is likely to be the case if the $\mathrm{BH}$ is scattered due to anisotropies of the galaxy, either when it is in isolation or during mergers.

\subsection{Dynamics of a seed black hole in its own galaxy}

We focus on a satellite galaxy which merges with the main galaxy when the age of the Universe is about 1 Gyr. In Fig. 5 we show snapshots at the beginning of the interaction between the main galaxy, on which the figure is centered, and the satellite, to the top left of the main galaxy. This satellite hosts a BH and we study its dynamics while the galaxy is in relative isolation. This case is interesting because it explores the prospects for a seed $\mathrm{BH}$ to remain surrounded by dense cold gas available for growing the $\mathrm{BH}$ and make it observable as a faint AGN.

We start by studying how the different sources of friction (dark matter, stars and gas) contribute to the dynamical evolution. Fig. 6 presents the density in gas and stars around the $\mathrm{BH}$ (we do not include dark matter since its contribution is negligible). Gas is more chaotic than stars, but stars themselves do not provide a constant acceleration because they are also irregularly distributed. Beyond the sheer inhomogeneity, gas can shock, cool, inflow and outflow making its dynamical friction contribution unpredictable a priori. The presence of satellites also perturbs the $\mathrm{BH}$ orbit when it is far from the center, see, e.g., Fig. 5: in a typical high-redshift environment a $\mathrm{BH}$ feels acceleration coming from different directions.

Moving to how this affects the BH's orbits, we show $\tau_{\mathrm{DF}}$ as a function of time in the bottom panel of Fig. 7, computed for the different simulations, using the method described in $\S 5.1 .3$. We also show in the top panel of Fig. 7 the distance of the $\mathrm{BH}$ to the center of its host galaxy.

Firstly, we find that, as long as the seeding mass of the $\mathrm{BH}$ is $10^{4} \mathrm{M}_{\odot}$, all the simulations, independently of the resolution and the different models used for the BH dynamics, show a similar trend: the sinking time is, at least, 1-10 Gyr. Since in all cases $v_{c}$ slowly increases from 7 to $30 \mathrm{~km} \mathrm{~s}^{-1}$ and $M_{\bullet}$ remains close to the BH seed mass, the reason of this large $\tau_{D F}$ is the dependency of the sinking time with the distance of the center of the galaxy, which is shown in the top panel of Fig. 7. Even in HR_PD_BH1e4 and HR_nPD_BH1e4, where the $\mathrm{BH}$ is 5 times heavier than the star particles (those mostly contributing to the dynamical friction here) and forms at $\sim 70 \mathrm{pc}$ from the centre, it is rapidly ejected and remains hundreds of pc away from the centre. Clumps and 


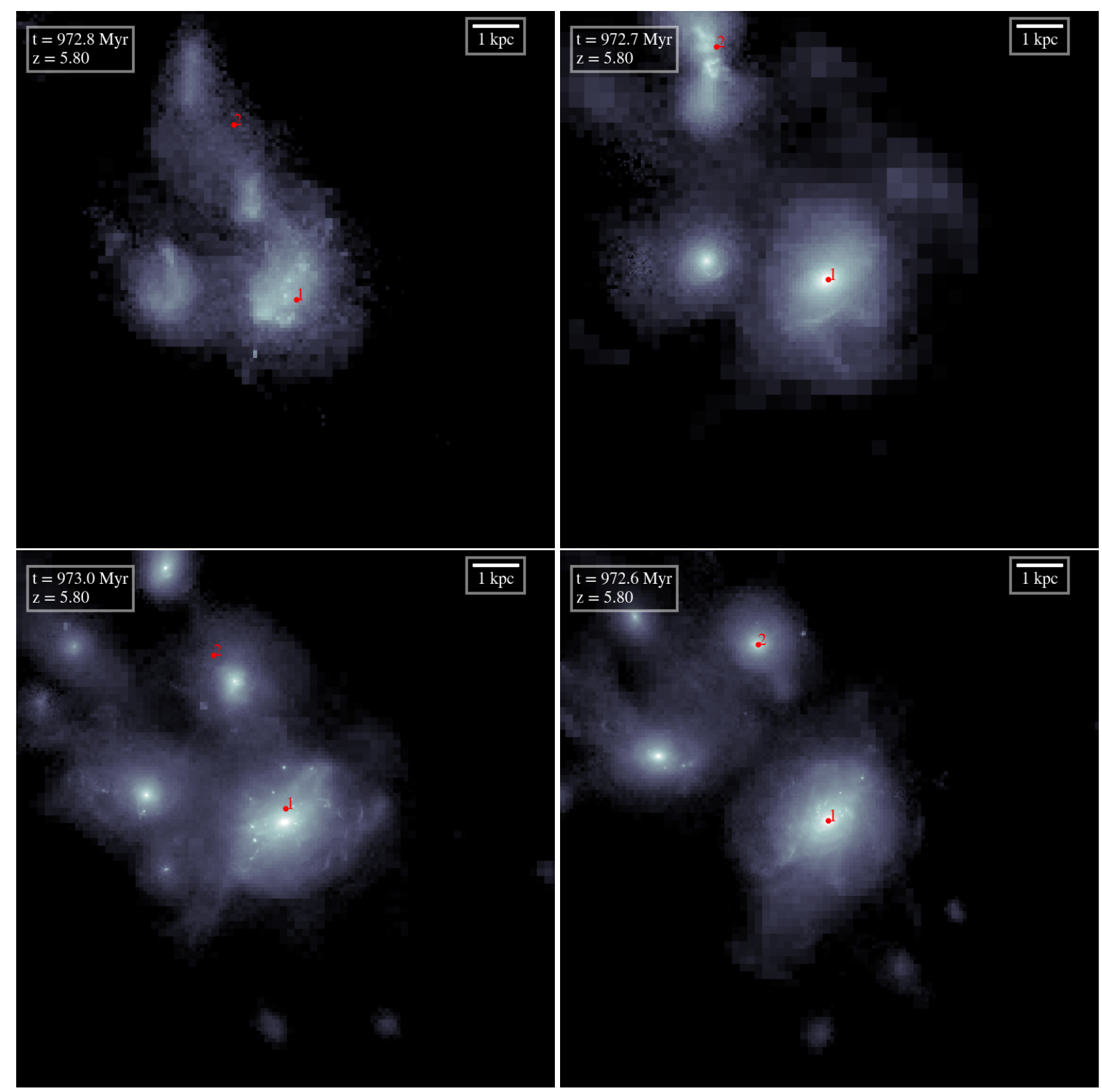

Figure 5. Top left: stellar density (black: $10^{-4} \mathrm{M}_{\odot} \mathrm{pc}^{-3}$, white: $1 \mathrm{M}_{\odot} \mathrm{pc}^{-3}$ ), centered on the main galaxy with a 80 comoving kpc box size, of LR_PD_BH1e4. Top right: MR_PD_BH1e4. Bottom left: HR_PD_BH1e4. Bottom right: HR_PD_BH1e5. The panels show the exact same galaxy at the same time to highlight the effects of resolution. In section 5.2 we discuss the dynamics of a $\mathrm{BH}$ in the satellite galaxy on the top-left corner of each panel (the BH is highlighted in red and its ID is 2 in the four panels). In section 5.3 we discuss instead the interaction between this $\mathrm{BH}$ and the main $\mathrm{BH}$ in the central galaxy (also highlighted in red, and its ID is 1 in the four panels).

anisotropies are observed both in the stellar and gas central distributions. Due to such irregularities in the underlying galaxy, the $\mathrm{BH}$ undergoes a physically-motivated random walk out of the centre of the potential well, as it also happens in lower redshift dwarfs (Bellovary et al. 2019). When the $\mathrm{BH}$ is more massive, $10^{5} \mathrm{M}_{\odot}$, it remains in the center of its host, with a sinking time less than $100 \mathrm{Myr} .10^{5} \mathrm{M}_{\odot}$ seems therefore to be the minimum requirement to imagine that a $\mathrm{BH}$ is well stabilized in the center of its host. BHs with masses lower than $10^{5} \mathrm{M}_{\odot}$ are scattered within the galaxy due to irregularities of the gas/stellar potential and oscillate around the center of their host galaxies, remaining far from the dense gas regions, therefore we expect them to have low accretion rates (Smith et al. 2018) and be difficult to observe.

\subsection{Formation of a black hole binary in a high-redshift galaxy merger}

We now focus on the same satellite galaxy, and follow the dynamical evolution of its BH during and after its host infalls into the halo of the larger galaxy. It is typically after 

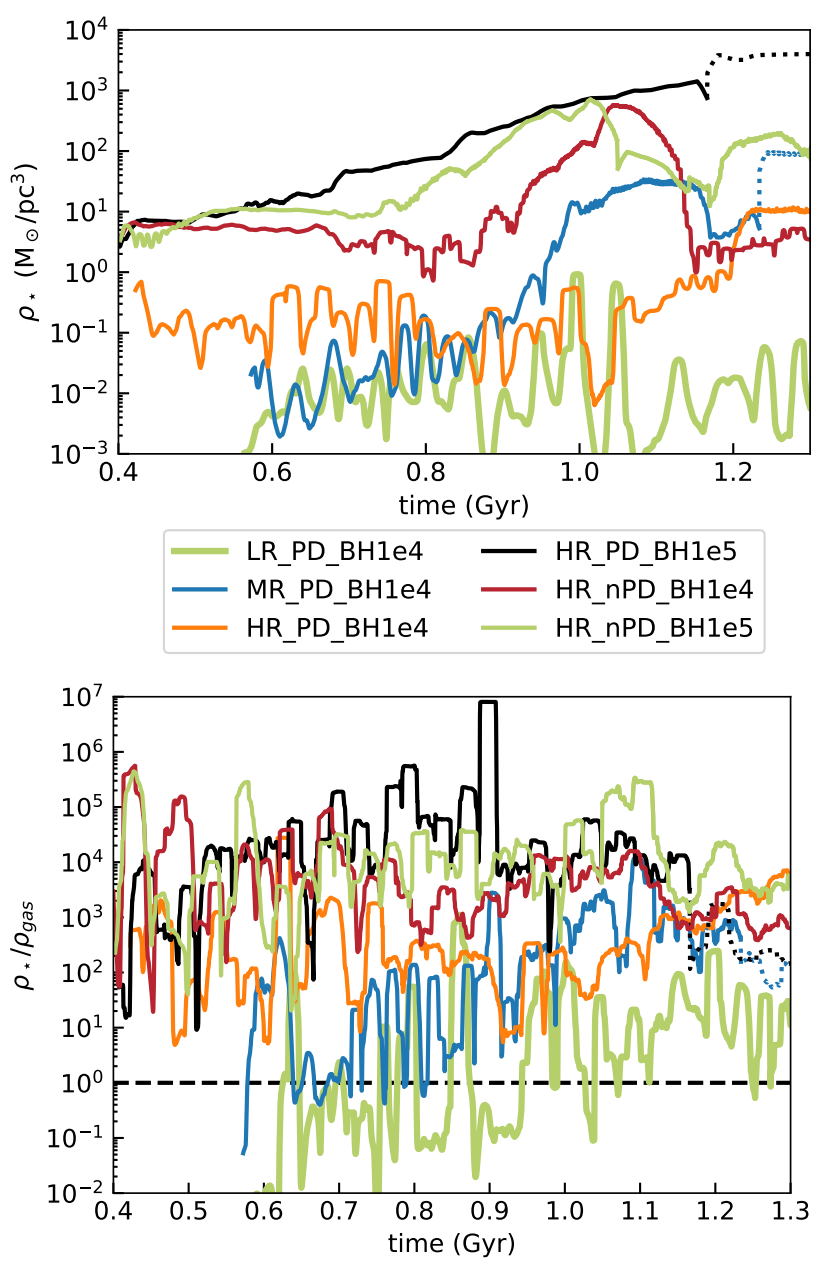

Figure 6. Top panel: mean stellar density within $4 \Delta x$ around the $\mathrm{BH}$ in the satellite galaxy, as a function of time for a subset of the simulations listed in Table 3, as noted in the inset. Bottom panel: ratio of the stellar density and gas density within $4 \Delta x$ around the satellite $\mathrm{BH}$, as a function of time.

this kind of event, when the galaxy remnant has settled and the massive BHs have sunk to the center of the potential well, that massive BH binaries form.

We show in Fig. $8 \tau_{\mathrm{DF}}$ as a function of time, for all the simulations (bottom panel). We see that, initially, when the $\mathrm{BH}$ is still embedded in the satellite galaxy (solid line), its dynamics is the same for all simulations: the large scale dynamics is independent of the subgrid model we use. However, what happens following the disruption of the satellite galaxy (dotted line) differs significantly from one simulation to the other: in some cases, the satellite BH sinks toward the center and "merges" (we recall that BHs are allowed to merge when they are separated by less than $4 \Delta x$ and the kinetic energy of the binary is lower than the gravitational energy, but the real merger happens below our resolution) with the central $\mathrm{BH}$ of the main galaxy (the subsequent evolution is shown as a dashed line), in other cases, the $\mathrm{BH}$ stalls hundreds of pc away from the center. We also show in the top panel of Fig. 8 the distance of the satellite $\mathrm{BH}$ to the central galaxy it is sinking in.

We first compare the simulations HR_PD_BH1e5 -
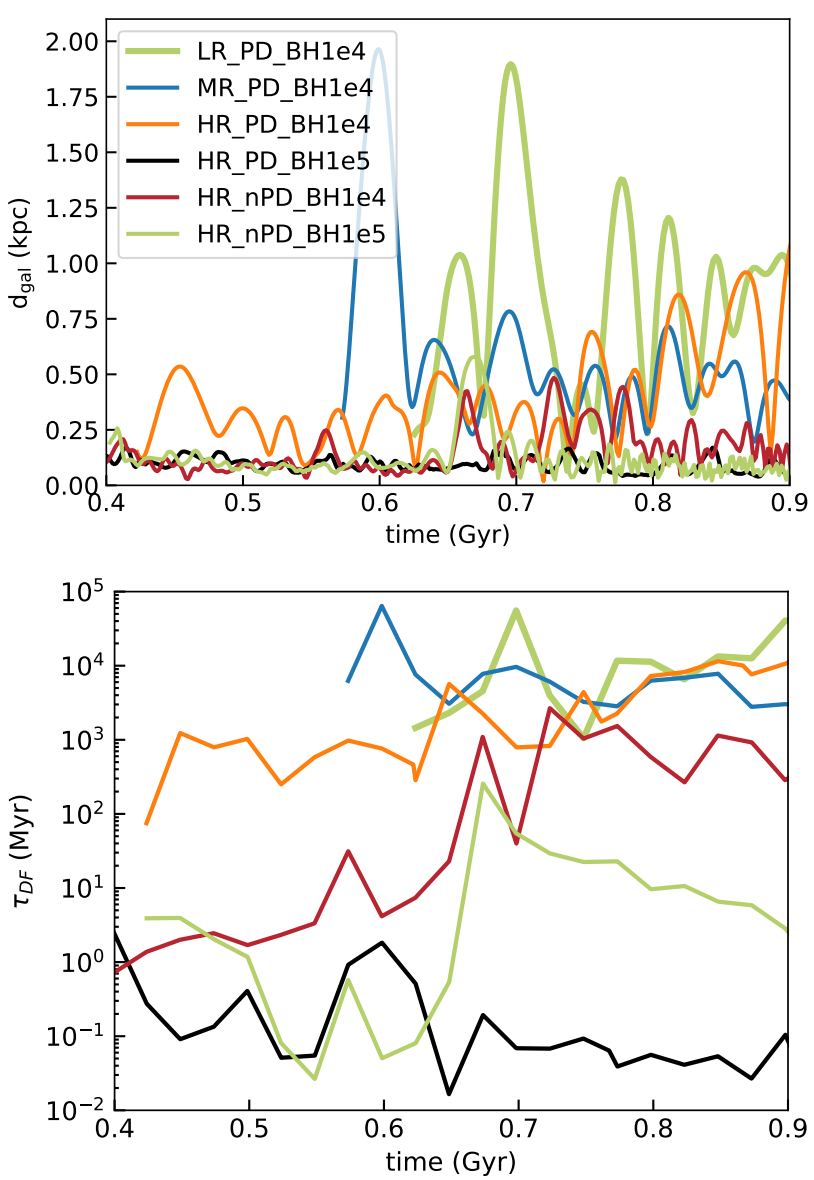

Figure 7. Top panel: distance of the $\mathrm{BH}$ from the center of its host galaxy, before interaction with another larger galaxy, as a function of time for all simulations listed in Table 3. Bottom panel: sinking time $\tau_{\mathrm{DF}}$ for the secondary $\mathrm{BH}$ with respect to its host galaxy, computed using Eq. (10) (replacing $M_{\boldsymbol{S}}$ by the mass of the $\mathrm{BH}$ in this equation), as a function of time.

HR_nPD_BH1e5, and HR_PD_BH1e4 - HR_nPD_BH1e4, which differ only by the use or not of our subgrid model for dynamical friction from stars and dark matter. Fig. 7 shows that the model does not help in keeping BHs in the center, as discussed in $\S 5.2$ : the galaxy is so chaotic that $\mathrm{BHs}$ wander no matter the implementation. When the galaxy is more settled, however, as it is the case when the satellite BH falls into the main galaxy, we see the effects of our model (see Fig. 8). When our prescription is used, the $\mathrm{BH}$ remains closer to the center; nonetheless the BHs do not merge as would happen if the BHs were artificially repositioned at the center of mass of the halo, as is sometimes done in cosmological simulations (e.g. Vogelsberger et al. 2013; Schaye et al. 2015).

We now focus on simulations with $10^{4} \mathrm{M}_{\odot}$ seeds. After its stellar and gaseous envelope has been dispersed (dotted line), the BH should take 1-100 Gyr to sink toward the center of the galaxy, and indeed, it stalls at $\sim$ hundreds of pc. This is in agreement with our understanding of dynamical friction: it is a very long process if the mass of the $\mathrm{BH}$ is low. The presence of a nuclear star cluster could speed-up the process (Biernacki et al. 2017), increasing the mass experiencing dynamical friction, but due to our limited resolution, 

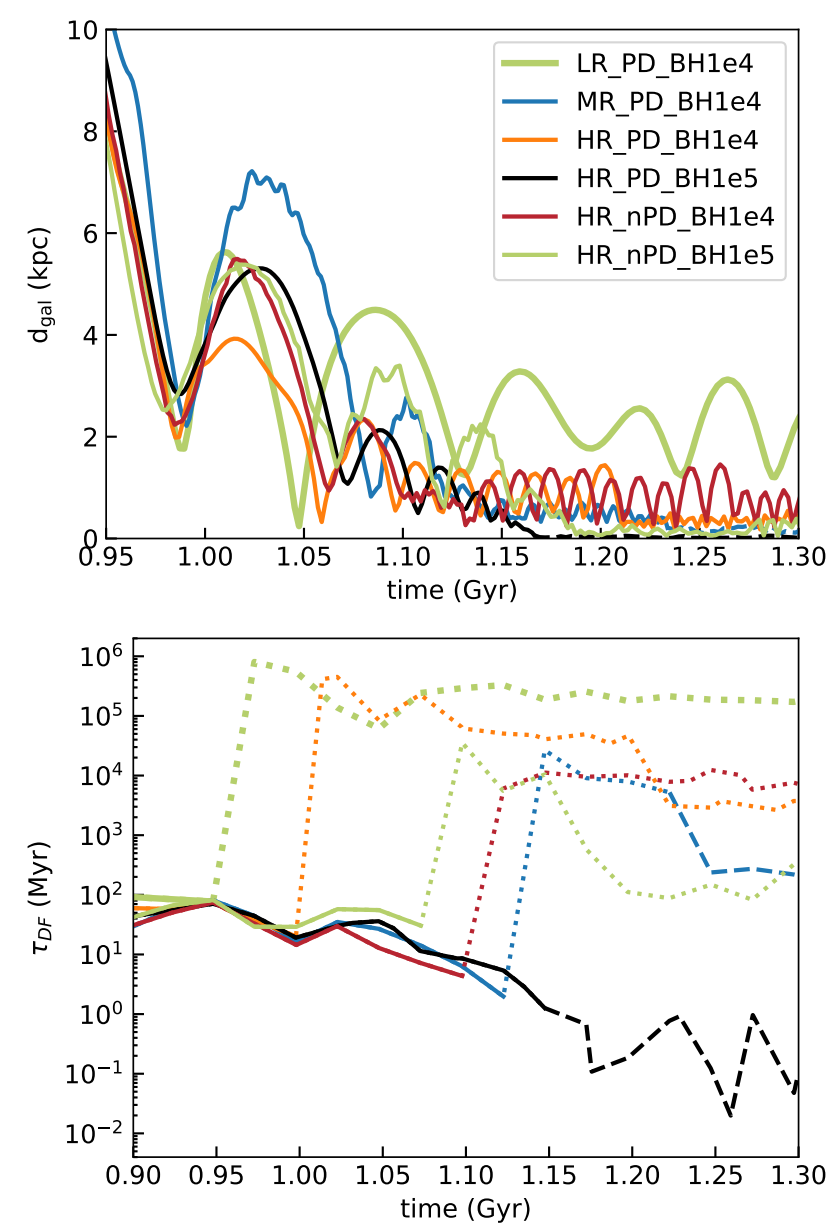

Figure 8. Top panel: distance of the $\mathrm{BH}$ originally in the satellite galaxy from the center of the main galaxy as a function of time for a subset of the simulations listed in Table 3, as noted in the inset. If the $\mathrm{BH}$ in the satellite galaxy merges with the $\mathrm{BH}$ of the central galaxy, we show its subsequent evolution with dashed lines. Bottom panel: sinking time $\tau_{\mathrm{DF}}$, computed using Eq. (10), as a function of time. We show the different phases: when the BH is still surrounded by material (solid line), when the BH is naked (dotted line; note the rapid increase in the sinking time because of the drop in $M_{S}$ in Eq. 10) and when the BH has merged with the $\mathrm{BH}$ of the central galaxy (dashed line).

such compact structures of typical size of a few pc to $\sim$ ten of pc are not captured here (Georgiev et al. 2016), and the envelope of the $\mathrm{BH}$ is rapidly stripped (dotted line). In the medium resolution case (MR_PD_BH1e4) the BH in the larger galaxy has also been scattered, similarly to what happened for the case studied in section 5.2. Accidentally, the two BHs merge while they are both off-center and the remnant of this merger remains hundreds of pc away from the center. If we admit that this merger is physical, it is interesting to note that mergers of light seeds BHs are possible, though the dynamics is highly erratic. Multiple BHs in galaxies, each inherited from a different merger, are generically expected (e.g. Governato et al. 1994; Schneider et al. 2002; Volonteri \& Perna 2005; Bonetti et al. 2018; Tremmel et al. 2018b).

Finally, we compare HR_PD_BH1e5 and HR_PD_BH1e4 which differ only by the seed mass of the BH. In
HR_PD_BH1e5, the BH being more massive, it remains surrounded by a dense stellar concentration which does not disrupt (no dotted line), increasing even more the effective $M_{S}$ and resulting in a smooth decay to the center of the main galaxy and a $\mathrm{BH}$ merger.

These experiments makes us believe that $<10^{4} \mathrm{M}_{\odot}$ seed $\mathrm{BHs}$ are less likely to contribute to the merging population observable by LISA than larger mass seed BHs. This does not exclude that these low-mass BHs may eventually sink in the center of galaxies and contribute to the massive $\mathrm{BH}$ population, but the presence of a dense stellar cluster or of bound gas on scales not resolved in this study, which would make the effective $M_{S}$ larger, appears to be crucial (e.g. Callegari et al. 2009). Off-center mergers, happening by chance, as in MR_nGB_PD_BH1e4 could also contribute.

\section{CONCLUSIONS}

We present a model to correct the dynamics of $\mathrm{BHs}$ in the RAMSES code, which is currently the only code to include a physically motivated model for dynamical friction onto BHs from gas, stars and dark matter. We use this model in a suite of cosmological simulations to understand the dynamics of seed $\mathrm{BHs}\left(10^{4}-10^{5} \mathrm{M}_{\odot}\right)$ in high-redshift galaxies and during galaxy mergers. We summarize our findings below:

- dynamical friction from stars has generically a more stabilizing effect than dynamical friction from gas, which can shock and is subject to inflows and outflows. In high-redshift galaxies, however, the stellar distribution is irregular and does not necessarily provide a smooth distribution within which BHs can decay undisturbed. The presence of satellite galaxies can also perturb the orbit of a $\mathrm{BH}$.

- From the results of our best resolution cosmological simulation, BHs with masses of the order of $10^{4} \mathrm{M}_{\odot}$ are subject to the fluctuations of the underlying stellar gravitational potential, which leads to a random walk-type of trajectory. This appears to be unique of a high- $z$ environment in which sub-structures undergo rapid evolution. If $\mathrm{BHs}$ were to be seeded in nuclear star clusters, or had masses of $10^{5} \mathrm{M}_{\odot}$ or higher, they would be well stabilized galaxy centres.

- Similarly, following galaxy mergers, if the mass of BHs in satellite galaxies is $\sim 10^{4} \mathrm{M}_{\odot}$, it is unlikely that they participate in the merging population, although off-center mergers can occur fortuitously. If seed BHs have larger masses, $\sim 10^{5} \mathrm{M}_{\odot}$, or they are embedded in dense bound stellar or gaseous envelopes, they can smoothly reach the center of the larger galaxy and merge with the companion $\mathrm{BH}$.

\section{ACKNOWLEDGMENTS}

MV and HP acknowledge support from the European Research Council (Project no. 614199, 'BLACK'). HP acknowledges support from the COST Association (CA16104 Gravitational waves, black holes and fundamental physics), and thanks the University of Milano Bicocca for hosting him. This work was granted access to the HPC resources of under the allocations A0020406955 and A0040406955 made by GENCI. This work has made use of the Horizon Cluster hosted by the Institut d'Astrophysique de Paris; we thank Stephane Rouberol for running smoothly this cluster for us. 
Finally, we acknowledge Darren Croton for his useful comments.

\section{REFERENCES}

Ahn J., Kim J., Shin J., Kim S., Choi Y.-Y., 2014, Journal of The Korean Astronomical Society, 47

Amaro-Seoane P., et al., 2017, preprint, (arXiv:1702.00786)

Antonini F., Merritt D., 2012, ApJ, 745, 83

Aubert D., Pichon C., Colombi S., 2004, MNRAS, 352, 376

Barausse E., 2012, MNRAS, 423, 2533

Beckmann R. S., et al., 2017, MNRAS, 472, 949

Begelman M. C., Blandford R. D., Rees M. J., 1980, Nature, 287, 307

Bellovary J. M., Cleary C. E., Munshi F., Tremmel M., Christensen C. R., Brooks A., Quinn T. R., 2019, MNRAS, 482, 2913

Biernacki P., Teyssier R., Bleuler A., 2017, MNRAS, 469, 295

Bonetti M., Haardt F., Sesana A., Barausse E., 2018, MNRAS, 477, 3910

Booth C. M., Schaye J., 2009, MNRAS, 398, 53

Bullock J. S., Dekel A., Kolatt T. S., Kravtsov A. V., Klypin A. A., Porciani C., Primack J. R., 2001, ApJ, 555, 240

Callegari S., Mayer L., Kazantzidis S., Colpi M., Governato F., Quinn T., Wadsley J., 2009, ApJ, 696, L89

Capelo P. R., Volonteri M., Dotti M., Bellovary J. M., Mayer L., Governato F., 2015, MNRAS, 447

Chandrasekhar S., 1943, ApJ, 97, 255

Chapon D., Mayer L., Teyssier R., 2013, MNRAS, 429, 3114

Colpi M., Mayer L., Governato F., 1999, ApJ, 525

Dayal P., Rossi E. M., Shiralilou B., Piana O., Choudhury T. R., Volonteri M., 2018, arXiv e-prints,

Dosopoulou F., Antonini F., 2017, ApJ, 840, 31

Dotti M., Colpi M., Haardt F., Mayer L., 2007, MNRAS, 379, 956

Dubois Y., Devriendt J., Slyz A., Teyssier R., 2012, MNRAS, 420, 2662

Dubois Y., Volonteri M., Silk J., 2014a, MNRAS, 440, 1590

Dubois Y., et al., 2014b, MNRAS, 444, 1453

Fakhouri O., Ma C.-P., Boylan-Kolchin M., 2010, MNRAS, 406, 2267

Georgiev I. Y., Böker T., Leigh N., Lützgendorf N., Neumayer N., 2016, MNRAS, 457, 2122

Goicovic F. G., Sesana A., Cuadra J., Stasyszyn F., 2016, preprint, (arXiv:1602.01966)

Governato F., Colpi M., Maraschi L., 1994, MNRAS, 271

Hahn O., Abel T., 2013, MUSIC: MUlti-Scale Initial Conditions, Astrophysics Source Code Library (ascl:1311.011)

Haiman Z., Kocsis B., Menou K., 2009, ApJ, 700, 1952

Hartwig T., Agarwal B., Regan J. A., 2018, MNRAS, 479, L23

Hernquist L., 1990, ApJ, 356, 359

Khan F. M., Berentzen I., Berczik P., Just A., Mayer L., Nitadori K., Callegari S., 2012, ApJ, 756, 30

Kimm T., Cen R., 2014, ApJ, 788, 121

Kormendy J., Ho L. C., 2013a, ARA\&A, 51, 511

Kormendy J., Ho L. C., 2013b, ARAA, 51, 511

Lacey C., Cole S., 1993, MNRAS, 262, 627

Mayer L., Kazantzidis S., Madau P., Colpi M., Quinn T., Wadsley J., 2007, Science, 316, 1874

Muñoz-Cuartas J. C., Macciò A. V., Gottlöber S., Dutton A. A., 2011, MNRAS, 411, 584

Navarro J. F., Frenk C. S., White S. D. M., 1997, ApJ, 490, 493

Ostriker E. C., 1999, ApJ, 513, 252

Perret V., 2016, DICE: Disk Initial Conditions Environment, Astrophysics Source Code Library (ascl:1607.002)

Pfister H., Lupi A., Capelo P. R., Volonteri M., Bellovary J. M., Dotti M., 2017, MNRAS, 471, 3646

Planck Collaboration et al., 2016, A\&A, 594, A13
Power C., Navarro J. F., Jenkins A., Frenk C. S., White S. D. M., Springel V., Stadel J., Quinn T., 2003, MNRAS, 338, 14

Quinlan G. D., 1996, New Astron., 1, 35

Rasera Y., Teyssier R., 2006, AAP, 445, 1

Reines A. E., Comastri A., 2016, Publ. Astron. Soc. Australia, $33, \mathrm{e} 054$

Ricarte A., Natarajan P., 2018, MNRAS, 481, 3278

Rosen A., Bregman J. N., 1995, ApJ, 440, 634

Schaye J., et al., 2015, MNRAS, 446, 521

Schneider R., Ferrara A., Natarajan P., Omukai K., 2002, ApJ, 571,30

Sesana A., Volonteri M., Haardt F., 2007a, MNRAS, 377, 1711

Sesana A., Haardt F., Madau P., 2007b, ApJ, 660, 546

Smith B., Regan J., Downes T., Norman M., O'Shea B., Wise J., 2018, preprint, (arXiv:1804.06477)

Sutherland R. S., Dopita M. A., 1993, APJS, 88, 253

Taffoni G., Mayer L., Colpi M., Governato F., 2003, MNRAS, 341,434

Teyssier R., 2002, AAP, 385, 337

Toro E. F., 1997, Riemann Solvers and Numerical Methods for Fluid Dynamics. Springer

Trebitsch M., Volonteri M., Dubois Y., Madau P., 2018, MNRAS, 478,5607

Tremmel M., Governato F., Volonteri M., Quinn T. R., 2015, MNRAS, 451, 1868

Tremmel M., Governato F., Volonteri M., Quinn T. R., Pontzen A., 2018a, MNRAS, 475, 4967

Tremmel M., Governato F., Volonteri M., Pontzen A., Quinn T. R., 2018b, ApJ, 857, L22

Tweed D., Devriendt J., Blaizot J., Colombi S., Slyz A., 2009, AAP, 506, 647

Vasiliev E., Antonini F., Merritt D., 2015, ApJ, 810, 49

Vogelsberger M., Genel S., Sijacki D., Torrey P., Springel V., Hernquist L., 2013, MNRAS, 436, 3031

Volonteri M., Perna R., 2005, MNRAS, 358, 913

Volonteri M., Haardt F., Madau P., 2003, ApJ, 582, 559

Woods T. E., et al., 2018, arXiv e-prints,

This paper has been typeset from a $\mathrm{TE}_{\mathrm{E}} \mathrm{X} / \mathrm{LATE} \mathrm{X}$ file prepared by the author. 